\title{
STRONGLY BOUNDED REPRESENTING MEASURES AND CONVERGENCE THEOREMS
}

\author{
IOANA GHENCIU \\ Mathematics Department, University of Wisconsin-River Falls, Wisconsin, 54022 \\ e-mail: ioana.ghenciu@uwrf.edu \\ and PAUL LEWIS \\ University of North Texas, Department of Mathematics, Box 311430 Denton, Texas, 76203-1430 \\ e-mail:lewis@unt.edu
}

(Received 9 March 2009; revised 4 September 2009; accepted 14 January 2010; first published online 22 March 2010)

\begin{abstract}
Let $K$ be a compact Hausdorff space, $X$ a Banach space and $C(K, X)$ the Banach space of all continuous functions $f: K \rightarrow X$ endowed with the supremum norm. In this paper we study weakly precompact operators defined on $C(K, X)$.

2010 Mathematics Subject Classification. Primary 46 E40, 46 G10; Secondary $46 \mathrm{~B} 20$.
\end{abstract}

1. Introduction. Suppose that $X$ and $Y$ are real Banach spaces, $K$ is a compact Hausdorff space, $C(K, X)$ is the Banach space of all continuous $X$-valued functions defined on $K$ (with the supremum norm) and $T: C(K, X) \rightarrow Y$ is an operator with representing measure $m: \Sigma \rightarrow L\left(X, Y^{* *}\right)$, where $\Sigma$ is the $\sigma$-algebra of subsets of $K$, $Y^{* *}$ is the bidual of $Y$ and $L\left(X, Y^{* *}\right)$ is the Banach space of all operators $T: X \rightarrow Y^{* *}$ [3]. Denote the semivariation of $m$ by $\tilde{m}$. The operator $T$ (or the measure $m$ ) is said to be strongly bounded if $\left(\tilde{m}\left(A_{i}\right)\right) \rightarrow 0$ whenever $\left(A_{i}\right)$ is a pairwise disjoint sequence from $\Sigma$. By Theorem 4.4 of [14], a strongly bounded representing measure takes its values in $L(X, Y)$. It is well known that if $T$ is unconditionally converging, then $m$ is strongly bounded $[3,19,28]$.

The Riesz Representation Theorem in this setting asserts that to each operator $T$ : $C(K, X) \rightarrow Y$ there corresponds a unique representing measure $m: \Sigma \rightarrow L\left(X, Y^{* *}\right)$ with finite semivariation so that $T(f)=\int_{K} f d m$ and $\|T\|=\tilde{m}(K)$. This correspondence between $T$ and $m$ will be denoted by $m \leftrightarrow T$. We note that [14] and Chapter 3 of [18] contain a detailed discussion of this setting. (The reader should note that for $f \in C(K, X), \int_{K} f d m \in Y$ even if $m$ is not $L(X, Y)$-valued.)

Let $\chi_{A}$ denote the characteristic function of a set $A$, and $B(\Sigma, X)$ denote the space of totally measurable functions on $\Sigma$ with values in $X$. Certainly $C(K, X)$ is contained isometrically in $B(\Sigma, X)$. Further, $B(\Sigma, X)$ embeds isometrically in $C(K, X)^{* *}$; e.g. see [14]. The reader should note that if $m \leftrightarrow T$, then $m(A) x=T^{* *}\left(\chi_{A} x\right)$, for each $A \in \Sigma$, $x \in X$. If $f \in B(\Sigma, X)$, then $f$ is the uniform limit of $X$-valued simple functions, $\int_{K} f d m$ is well defined, which defines an extension $\hat{T}$ of $T$; e.g. see [18]. Theorem 2 of [7] shows that $\hat{T}$ maps $B(\Sigma, X)$ into $Y$ if and only if the representing measure $m$ of $T$ is $L(X, Y)$ valued. If $T: C(K, X) \rightarrow Y$ is strongly bounded, then $m$ is $L(X, Y)$-valued [14], and thus $\hat{T}: B(\Sigma, X) \rightarrow Y$. Since $\hat{T}$ is the restriction to $B(\Sigma, X)$ of the operator $T^{* *}$, it is 
clear that an operator $T: C(K, X) \rightarrow Y$ is compact (resp. weakly compact) if and only if its extension $\hat{T}: B(\Sigma, X) \rightarrow Y$ is compact (resp. weakly compact). Several authors have found the study of $\hat{T}$ to be quite helpful. We mention the work of Batt and Berg [7], Bombal and Cembranos [13] and Bombal and Porras [11]. In these papers it has been proved that if $m$ is strongly bounded, then $T: C(K, X) \rightarrow Y$ is weakly compact, compact, Dunford-Pettis, Dieudonné, unconditionally converging, strictly singular or strictly cosingular if and only if its extension $\hat{T}: B(\Sigma, X) \rightarrow Y$ has the same property. Our results will be concerned with relating properties of the operator $T$ to properties of its representing measure in the case of weakly precompact operators and operators with weakly precompact adjoints. An operator $T: X \rightarrow Y$ is called weakly precompact (or almost weakly compact) if every sequence in the image of a bounded set has a weakly Cauchy subsequence.

The general bilinear integral of Bartle [4] can be used in the context of strongly bounded representing measures to establish convergence results which unify several approaches and have numerous applications and corollaries. Although the convergence theorems in [4] are similar to some of the conclusions in our first theorem, it is not clear that [4] can be used to obtain the specific results we desire. For this reason, as well as for the convenience of the reader, we include a brief description of the bilinear integral we shall use and a proof of the convergence results we need. In the process, the technique and the results in [21] are extended.

Suppose that $m$ is a strongly bounded representing measure with control measure $\lambda$, i.e. $0 \leq \lambda \in \operatorname{rca}(\Sigma)$ and $\tilde{m}(A) \rightarrow 0$ as $\lambda(A) \rightarrow 0$. If $g \in L^{1}(\lambda, X)$ and $g$ is pointwise bounded, choose a uniformly pointwise bounded sequence of $X$-valued simple functions $\left(s_{n}\right)$ so that $s_{n}(t) \rightarrow g(t)$ a.e.- $\lambda$ (see [20], p. 117). The standard approach in Section 7, pp. 106-108, of Dinculeanu [18] is used to define the integral of an $X$-valued simple function with respect to an $L(X, Y)$-valued measure with finite semivariation, i.e. if $s=\sum \chi_{A_{i}} x_{i}$ and $m: \Sigma \rightarrow L(X, Y)$ is finitely additive and has finite semivariation, then $\int s d m$ is defined to be $\sum m\left(A_{i}\right) x_{i}$. Egoroff's theorem guarantees that $\left(\int_{K} s_{n} d m\right)$ converges. Define $\int_{K} g d m$ to be $\lim _{n} \int_{K} s_{n} d m$. It is not difficult to check that $\int_{K} g d m$ is well defined.

\section{Main results.}

Theorem 1. Suppose that $m \leftrightarrow T: C(K, X) \rightarrow Y$ is strongly bounded and $\lambda$ is a control measure for $m$.

(i) If $\left(g_{n}\right)$ is a uniformly pointwise bounded sequence and $\left(g_{n}\right) \rightarrow 0$ in $L^{1}(\lambda, X)$, then $\left(\int_{K} g_{n} d m\right) \rightarrow 0$ in $Y$. Consequently, if $\left(g_{n}\right)$ is uniformly pointwise bounded and $\left(g_{n}\right) \stackrel{w}{\rightarrow} 0$, then $\left(\int_{K} g_{n} d m\right) \stackrel{w}{\rightarrow} 0$.

(ii) If $\left(h_{n}\right)$ is a uniformly pointwise bounded sequence in $L^{1}(\lambda, X)$ and $\left(h_{n}(t)\right)$ is weakly Cauchy for each $t \in K$, then $\left(\int_{K} h_{n} d m\right)$ is weakly Cauchy in $Y$.

(iii) Suppose that $H$ is a bounded set in $C(K, X)$. If $\left(f_{n}\right)$ is a sequence in $H$ and $f: K \rightarrow X$ is a function such that $f_{n}(t) \rightarrow f(t)$ for each $t \in K$, then $\left(\int_{K} f_{n} d m\right) \rightarrow \int_{K} f d m$.

Proof. (i) Without loss of generality, suppose that $\left\|g_{n}(t)\right\|<1$ for all $n \in \mathbb{N}$ and all $t \in K$. Since $\left(\int_{K}\left\|g_{n}\right\| d \lambda\right) \rightarrow 0$, we may suppose without loss of generality that $\left(g_{n}(t)\right) \rightarrow 0$ for almost all $t \in K$. Let $\epsilon>0$ and choose $E \in \Sigma$ such that $\tilde{m}(K \backslash E)<\epsilon$ and $\left(g_{n}\right) \rightarrow 0$ uniformly on $E$. Choose $n_{0} \in \mathbb{N}$ so that if $n \geq n_{0}$, then $\left\|g_{n}(t)\right\| \leq \epsilon, t \in E$. 
The definition of $\int_{E} g_{n} d m$ and $\int_{K \backslash E} g_{n} d m$ show that for $n \geq n_{0}$,

$$
\left\|\int_{K} g_{n} d m\right\|=\left\|\int_{E} g_{n} d m+\int_{K \backslash E} g_{n} d m\right\| \leq \epsilon \tilde{m}(E)+\epsilon .
$$

We claim that $\left(\int_{K} g_{n} d m\right) \stackrel{w}{\rightarrow} 0$, when $\left(g_{n}\right)$ is uniformly pointwise bounded and $\left(g_{n}\right) \stackrel{w}{\rightarrow} 0$ in $L^{1}(\lambda, X)$. Indeed, if $\left(g_{n_{i}}\right)$ is an arbitrary subsequence of $\left(g_{n}\right)$, then $0 \in \overline{c o}\left\{g_{n_{i}}: i \geq\right.$ $1\}$ (since $\left(g_{n}\right) \stackrel{w}{\rightarrow} 0$ ). Thus, $0 \in \overline{c o}\left\{\int_{K} g_{n_{i}} d m: i \geq 1\right\}$. This implies that $\left(\int_{K} g_{n} d m\right) \stackrel{w}{\rightarrow} 0$. Otherwise, one can strictly separate 0 from the closed convex hull of some subsequence of $\left(\int_{K} g_{n} d m\right)$, a contradiction.

(ii) Without loss of generality, suppose $\left\|h_{n}(t)\right\|<1$ for all $n \in \mathbb{N}$ and $t \in K$. Let $\epsilon>0$. Using the existence of a control measure for $m$ and Lusin's theorem, we can find a compact subset $K_{0}$ of $K$ such that $\tilde{m}\left(K \backslash K_{0}\right)<\epsilon / 2$ and $\phi_{n}=\left.h_{n}\right|_{K_{0}}$ is continuous for each $n \in \mathbb{N}$. Let $H=\left[\phi_{n}\right]$ be the closed linear span of $\left(\phi_{n}\right)$ in $C\left(K_{0}, X\right)$ and $S$ : $H \rightarrow C(K, X)$ be the isometric extension operator given by Theorem 1 of [13]. Let $\psi_{n}=S\left(\phi_{n}\right), n \in \mathbb{N}$. Since $\left(\phi_{n}(t)\right)$ is weakly Cauchy for each $t \in K_{0}$, the sequence $\left(\phi_{n}\right)$ is weakly Cauchy in $C\left(K_{0}, X\right)$ (Theorem 9 of [19], Lemma 3.2 of [3]). Then $\left(\psi_{n}\right)$ is weakly Cauchy in $C(K, X)$ and $\left(T\left(\psi_{n}\right)\right)$ is weakly Cauchy. For each $n \in \mathbb{N}$,

$$
\left\|\int_{K} h_{n} d m-\int_{K} \psi_{n} d m\right\|=\left\|\int_{K \backslash K_{0}}\left(h_{n}-\psi_{n}\right) d m\right\| \leq 2 \tilde{m}\left(K \backslash K_{0}\right)<\epsilon .
$$

Then $\left(\int_{K} h_{n} d m\right)$ is weakly Cauchy.

(iii) Let $H$ be the unit ball of $C(K, X),\left(f_{n}\right)$ be a sequence in $H$ and let $f: K \rightarrow X$ be a function such that $f_{n}(t) \rightarrow f(t)$ for each $t \in K$. Without loss of generality suppose that $\|f(t)\| \leq 1, t \in K$. Then $f$ is strongly measurable (by the Pettis measurability theorem) and $\int_{K} f d m$ exists. Let $\epsilon>0$. Use Lusin's theorem and the existence of the control measure to choose a compact subset $K_{0}$ of $K$ such that $g=\left.f\right|_{K_{0}}$ is continuous and $\tilde{m}\left(K \backslash K_{0}\right)<\epsilon / 2$. Let $g_{n}=\left.f_{n}\right|_{K_{0}}, n \in \mathbb{N}$. Use Egoroff's theorem to choose a compact subset $K_{1}$ of $K_{0}$ such that $\tilde{m}\left(K_{0} \backslash K_{1}\right)<\epsilon / 2$ and $\left(g_{n}-g\right) \rightarrow 0$ uniformly on $K_{1}$. Let $n_{0} \in \mathbb{N}$ so that if $n \geq n_{0}$, then $\left\|g_{n}(t)-g(t)\right\| \leq \epsilon, t \in K_{1}$. For $n \geq n_{0}$, we have

$$
\begin{aligned}
\left\|\int_{K}\left(f_{n}-f\right) d m\right\| & =\left\|\int_{K_{1}}\left(f_{n}-f\right) d m+\int_{K_{0} \backslash K_{1}}\left(f_{n}-f\right) d m+\int_{K \backslash K_{0}}\left(f_{n}-f\right) d m\right\| \\
& \leq \sup _{t \in K_{1}}\left\|g_{n}(t)-g(t)\right\| \tilde{m}\left(K_{1}\right)+2 \tilde{m}\left(K_{0} \backslash K_{1}\right)+2 \tilde{m}\left(K \backslash K_{0}\right) \\
& \leq \epsilon \tilde{m}(K)+2 \epsilon .
\end{aligned}
$$

Abbott [1] gave an example of a pair $m \leftrightarrow T$ such that $T$ is weakly precompact and $m$ is not strongly bounded. The following corollary is related to results in [32].

COROLlaRY 2. If $\ell_{1} \hookrightarrow X$, then every strongly bounded operator $T: C(K, X) \rightarrow Y$ is weakly precompact.

Proof. Suppose that $T: C(K, X) \rightarrow Y$ is a strongly bounded operator with representing measure $m$ and control measure $\lambda$. We have

$$
T(f)=\int_{K} f d m, f \in C(K, X) .
$$


Let $\left(f_{n}\right)$ be a sequence in the unit ball of $C(K, X)$. Then $\left(f_{n}\right)$ is uniformly integrable in $L^{1}(\lambda, X)$. Since $\ell_{1} \hookrightarrow X,\left(f_{n}\right)$ is weakly precompact in $L^{1}(\lambda, X)$ [12]. Without loss of generality, suppose $\left(f_{n}\right)$ is weakly Cauchy in $L^{1}(\lambda, X)$. By results of Talagrand [30], we can write $f_{n}=g_{n}+h_{n}$ a.e. in $K$, where $\left(g_{n}\right)$ and $\left(h_{n}\right)$ are sequences in $L^{1}(\lambda, X)$ such that $\left(g_{n}\right)$ is weakly null in $L^{1}(\lambda, X)$ and $\left(h_{n}(t)\right)$ is weakly Cauchy for each $t \in K$. Further, we have $\left(g_{n}\right)$ and $\left(h_{n}\right)$ uniformly pointwise bounded. By Theorem $1,\left(\int_{K} g_{n} d m\right) \stackrel{w}{\rightarrow} 0$ and $\left(\int_{K} h_{n} d m\right)$ is weakly Cauchy. Hence $\left(\int_{K} f_{n} d m\right)$ is weakly Cauchy, and thus $T$ is weakly precompact.

3. Applications. An operator $T: X \rightarrow Y$ is called a Dieudonné (or weakly completely continuous) operator if $T$ maps weakly Cauchy sequences in $X$ to weakly convergent sequences in $Y$, and $X$ is said to have the Dieudonné property if every Dieudonné operator with domain $X$ is weakly compact [25]. If $X$ is a $C(K)$-space or if $\ell_{1} \leftrightarrow X$, then $X$ has the Dieudonné property.

Corollary 3. ([21, 26]) If $\ell_{1} \hookrightarrow X$, then $C(K, X)$ has the Dieudonné property.

Proof. If $m \leftrightarrow T: C(K, X) \rightarrow Y$ is a Dieudonné operator, then $T$ is unconditionally converging and $m$ is strongly bounded $[\mathbf{3}, \mathbf{1 9}, \mathbf{2 8}]$. Let $\left(f_{n}\right)$ be a sequence in the unit ball of $C(K, X)$. Using the arguments in Corollary 2 and Theorem 1, we obtain sequences $\left(g_{n}\right),\left(h_{n}\right)$ and $\left(\psi_{n}\right)$ so that

$$
T\left(f_{n}\right)=\int_{K} f_{n} d m=\int_{K} g_{n} d m+\int_{K} h_{n} d m,
$$

$\left(\int_{K} g_{n} d m\right) \stackrel{w}{\rightarrow} 0,\left(\psi_{n}\right)$ is weakly Cauchy in $C(K, X)$ and $\left\|\int_{K} h_{n} d m-T\left(\psi_{n}\right)\right\| \rightarrow 0$. Let $y \in Y$ such that $\left(T\left(\psi_{n}\right)\right) \stackrel{w}{\rightarrow} y$. Then $\left(\int_{K} h_{n} d m\right) \stackrel{w}{\rightarrow} y$, and thus $\left(T\left(f_{n}\right)\right) \stackrel{w}{\rightarrow} y$.

A Banach space $X$ has property $(u)$ if for every weakly Cauchy sequence $\left(x_{n}\right)$ in $X$, there is a weakly unconditionally converging series $\sum y_{n}$ in $X$ such that $\left(x_{n}-\right.$ $\left.\sum_{i=1}^{n} y_{i}\right) \stackrel{w}{\rightarrow} 0$. A Banach space $X$ has property $(V)$ if every unconditionally converging operator $T$ from $X$ to any Banach space $Y$ is weakly compact [27].

COROLlaRY 4. (i) $([14,32])$ If $X$ is reflexive, then every strongly bounded operator $T: C(K, X) \rightarrow Y$ is weakly compact.

(ii) ([27]) If $X$ is reflexive, then $C(K, X)$ has property $(V)$.

(iii) $([15,32])$ If $\ell_{1} \hookrightarrow X$ and $X$ has property $(u)$, then $C(K, X)$ has property $(V)$.

Proof. (i) Let $m \leftrightarrow T: C(K, X) \rightarrow Y$ be a strongly bounded operator and $\left(f_{n}\right)$ be a sequence in the unit ball of $C(K, X)$. Repeating the construction in Corollary 2, we obtain uniformly pointwise bounded sequences $\left(g_{n}\right),\left(h_{n}\right)$ in $L^{1}(\lambda, X)$ so that $f_{n}=$ $g_{n}+h_{n}$ a.e. in $K,\left(g_{n}\right)$ is weakly null in $L^{1}(\lambda, X)$ and $\left(h_{n}(t)\right)$ is weakly Cauchy for each $t \in K$. Let $\epsilon>0$. Repeating the construction in Theorem 1, we obtain a compact subset $K_{0}$ of $K$ and a sequence $\left(\phi_{n}\right)$ so that $\tilde{m}\left(K \backslash K_{0}\right)<\epsilon / 2$ and $\phi_{n}=\left.h_{n}\right|_{K_{0}}$ is continuous for each $n \in \mathbb{N}$; further, $\left(\phi_{n}(t)\right)$ is weakly Cauchy for each $t \in K_{0},\left(\int_{K} g_{n} d m\right) \stackrel{w}{\rightarrow} 0$ and

$$
T\left(f_{n}\right)=\int_{K} f_{n} d m=\int_{K} g_{n} d m+\int_{K} h_{n} d m
$$

Let $\phi: K_{0} \rightarrow X$ be a function so that $\left(\phi_{n}(t)\right) \stackrel{w}{\rightarrow} \phi(t), t \in K_{0}$ (the reflexivity of $X$ assures the existence of $\phi)$. Then $\phi$ is bounded, and since for each $n, \phi_{n}$ is continuous, $\phi$ 
is separably valued and weakly measurable. By Pettis's measurability theorem, $\phi$ is strongly measurable. Use Lusin's theorem and the existence of the control measure to choose a compact subset $K_{1}$ of $K_{0}$ such that $h=\left.\phi\right|_{K_{1}}$ is continuous and $\tilde{m}\left(K_{0} \backslash K_{1}\right)<$ $\epsilon / 2$. Hence $\left(\phi_{n}\right) \stackrel{w}{\rightarrow} h$ in $C\left(K_{1}, X\right)$.

Let $H=\left[\phi_{n}\right]$ be the closed linear span of $\left(\phi_{n}\right)$ in $C\left(K_{1}, X\right)$ and $S: H \rightarrow C(K, X)$ be the isometric extension operator given by Theorem 1 of [13]. Let $\psi_{n}=S\left(\phi_{n}\right)$, $n \in \mathbb{N}$ and $\psi=S(h)$. Since $\left(\phi_{n}\right) \stackrel{w}{\rightarrow} h$ in $C\left(K_{1}, X\right)$, it follows that $\left(T S\left(\phi_{n}\right)\right) \stackrel{w}{\rightarrow} T S(h)$; i.e. $\left(T\left(\psi_{n}\right)\right) \stackrel{w}{\rightarrow} T(\psi):=y$ in $Y$. Further, for each $n \in \mathbb{N}$,

$$
\left\|\int_{K} h_{n} d m-\int_{K} \psi_{n} d m\right\|=\left\|\int_{K \backslash K_{1}}\left(h_{n}-\psi_{n}\right) d m\right\| \leq 2 \tilde{m}\left(K \backslash K_{1}\right)<2 \epsilon .
$$

Then $\left(\int_{K} h_{n} d m\right) \stackrel{w}{\rightarrow} y$, hence $\left(\int_{K} f_{n} d m\right) \stackrel{w}{\rightarrow} y$.

(ii) Every unconditionally converging operator on $C(K, X)$ is strongly bounded [3, 19, 28], and thus weakly compact.

(iii) If $T: C(K, X) \rightarrow Y$ is an unconditionally converging operator, then $T$ is a Dieudonné operator, since $X$ has property $(u)$ [32]. By Corollary 3, $T$ is weakly compact.

Gamlen [23] proved that if $X^{*}$ has the Radon-Nikodym property and $Y$ is weakly sequentially complete, then any operator $T: C(K, X) \rightarrow Y$ is weakly compact. Bello [8] generalized this result to the case of $X$ not containing copies of $\ell_{1}$. The following result contains Theorem 12 [8].

COROLlaRY 5. Suppose that $\ell_{1} \nrightarrow X$.

(i) If $c_{0} \hookrightarrow Y$, then every operator $T: C(K, X) \rightarrow Y$ is weakly precompact.

(ii) If $Y$ is weakly sequentially complete, then every operator $T: C(K, X) \rightarrow Y$ is weakly compact.

(iii) If $Y$ has the Schur property, then every operator $T: C(K, X) \rightarrow Y$ is compact.

Proof. (i) Suppose $T: C(K, X) \rightarrow Y$ is an operator. Since $c_{0} \hookrightarrow Y, T$ is unconditionally converging, and thus strongly bounded $[3,19,28]$. By Corollary 2 , $T$ is weakly precompact.

(ii) Since $Y$ is weakly sequentially complete, $T$ is weakly compact.

(iii) Since $Y$ has the Schur property, $T$ is compact.

We remark that if $c_{0} \hookrightarrow Y$ and $T: C(K, X) \rightarrow Y$ is an operator with representing measure $m$, then $m$ is countably additive. To see this, note that $T$ is unconditionally converging, $m$ is strongly bounded, and thus countably additive [3, 14].

COROLlary 6. (i) If $X^{*}$ has the Radon-Nikodym property, then every strongly bounded operator $T: C(K, X) \rightarrow Y$ is weakly precompact.

(ii) If $X^{*}$ is separable, then every strongly bounded operator $T: C(K, X) \rightarrow Y$ is weakly precompact.

Proof. (i) If $X^{*}$ has the Radon-Nikodym property, then $\ell_{1} \hookrightarrow X$ [17]. Apply Corollary 2. (ii) If $X^{*}$ is separable, then $X^{*}$ has the Radon-Nikodym property.

Corollary 7. Suppose that $X$ is a Banach space such that for every compact Hausdorff space $K$ and every Banach space $Y$, an operator $m \leftrightarrow T: C(K, X) \rightarrow Y$ is weakly precompact whenever $m$ satisfies the following conditions: 
(i) $m$ is strongly bounded and

(ii) $m(A): X \rightarrow Y$ is weakly precompact for each $A \in \Sigma$.

Then $\ell_{1}$ is not complemented in $X$.

Proof. Suppose that $\ell_{1}$ is complemented in $X$. If $P: X \rightarrow \ell_{1}$ is a projection, then $P$ is not compact. By Theorem 2.2 of [2], there is a compact space $\Delta$ and a continuous linear surjection $m \leftrightarrow T: C(\Delta, X) \rightarrow \ell_{1}$ so that $m$ is strongly bounded and $m(A)$ : $X \rightarrow Y$ is compact for each $A \in \Sigma$. Since $T$ is a surjection onto $\ell_{1}, T$ is not weakly precompact.

COROLlary 8. If $\ell_{1} \hookrightarrow X^{*}$ and $T: C(K, X) \rightarrow Y$ is strongly bounded, then $T$ and $T^{*}$ are weakly precompact.

Proof. $T^{*}$ is weakly precompact by Theorem 9 [6]. Since $\ell_{1} \hookrightarrow X^{*}, \ell_{1} \hookrightarrow X([16]$, p. 211). Apply Corollary 2.

Corollary 9. Suppose that $\ell_{1} \hookrightarrow X^{*}$ and $T: C(K, X) \rightarrow Y$ is an operator. Then the following are equivalent:

(i) $T$ is strongly bounded.

(ii) $T^{*}$ is weakly precompact.

(iii) $T$ is unconditionally converging.

Proof. (i) implies (ii). If $T: C(K, X) \rightarrow Y$ is a strongly bounded operator, then $T^{*}$ is weakly precompact by Theorem $9[6]$.

(ii) implies (iii). If $T^{*}$ is weakly precompact, then $T$ is unconditionally converging by Corollary 2 [6].

(iii) implies (i). Every unconditionally converging operator on $C(K, X)$ is strongly bounded $[3,19,28]$.

COROLlaRY 10. ([14]) If $c_{0} \hookrightarrow X$ and $T: C(K, X) \rightarrow Y$ is a strongly bounded operator, then $T$ is unconditionally converging.

Proof. It is enough to show that if $\sum f_{n}$ is weakly unconditionally converging in $C(K, X)$, then $\left\|T\left(f_{n}\right)\right\| \rightarrow 0$. Suppose that $\sum f_{n}$ is weakly unconditionally converging. Then for each $t \in K, \sum f_{n}(t)$ is weakly unconditionally converging, and thus unconditionally converging in $X$ (since $c_{0} \hookrightarrow X$ ). Hence $\left\|f_{n}(t)\right\| \rightarrow 0$ for each $t \in K$, and $\left(T\left(f_{n}\right)\right) \rightarrow 0$ by Theorem 1 .

An operator $T: X \rightarrow Y$ is called completely continuous (or Dunford-Pettis) if $T$ maps weakly Cauchy sequences to norm convergent sequences. The Banach space $X$ has the Dunford-Pettis property (DPP) if every weakly compact operator with domain $X$ is completely continuous. Talagrand showed that there is a Banach space $X$ such that $X^{*}$ has the Schur property (hence $X$ has the DPP), but neither $C(K, X)$ nor $L^{1}\left(X^{*}\right)$ has the DPP [31].

COROLLARY 11. Suppose that $X$ has the Schur property. Then the following assertions hold:

(i) Every strongly bounded operator $T: C(K, X) \rightarrow Y$ is completely continuous. 
(ii) ([19]) $C(K, X)$ has the DPP.

(iii) ([14]) If $c_{0} \hookrightarrow Y$, then every operator $T: C(K, X) \rightarrow Y$ is completely continuous.

(iv) If $T: C(K, X) \rightarrow Y$ is an operator with a weakly precompact adjoint, then $T$ is completely continuous.

(v) If $T: C(K, X) \rightarrow Y$ is an operator, then $T$ is a Dieudonné operator if and only if $T$ is completely continuous.

Proof. (i) Let $\left(f_{n}\right)$ be a weakly null sequence in the unit ball of $C(K, X)$ and $T$ : $C(K, X) \rightarrow Y$ be a strongly bounded operator. Since $\left(f_{n}(t)\right)$ is weakly null in $X$, and $X$ has the Schur property, $\left\|f_{n}(t)\right\| \rightarrow 0$ for each $t \in K$. By Theorem $1,\left(T\left(f_{n}\right)\right) \rightarrow 0$, and thus $T$ is completely continuous.

(ii) Every weakly compact operator $T: C(K, X) \rightarrow Y$ is strongly bounded [14]. Then $T$ is completely continuous, and thus $C(K, X)$ has the DPP.

(iii) If $c_{0} \hookrightarrow Y$ and $T: C(K, X) \rightarrow Y$ is an operator, then $T$ is unconditionally converging, and thus strongly bounded $[3,19,28]$. By part (i), $T$ is completely continuous.

(iv) If $T^{*}: Y^{*} \rightarrow C(K, X)^{*}$ is weakly precompact, then $T: C(K, X) \rightarrow Y$ is unconditionally converging (Corollary 2 in [6]), and thus strongly bounded. Apply (i).

(v) If $T: C(K, X) \rightarrow Y$ is a Dieudonné operator, then $T$ is unconditionally converging, hence strongly bounded. Apply (i). The converse is clear.

The next result establishes a connection between weakly precompact operators and unconditionally converging adjoints. It is known that if $T: X \rightarrow Y$ is an operator, then $T\left(B_{X}\right)$ is a $V^{*}$-subset of $Y$ if and only if $T^{*}: Y^{*} \rightarrow X^{*}$ is unconditionally converging $[5,24]$.

THEOREM 12. If $T: X \rightarrow Y$ is weakly precompact, then $T^{*}: Y^{*} \rightarrow X^{*}$ is unconditionally converging.

Proof. Suppose $T: X \rightarrow Y$ is weakly precompact. Then $T\left(B_{X}\right)$ is weakly precompact, and thus a $V^{*}$-subset of $Y$ [27]. It follows that $T^{*}$ is unconditionally converging.

We remark that the converse of this theorem is not true. Specifically, let $X$ be a Banach space such that $\ell_{1} \hookrightarrow X$ and $\ell_{1} \stackrel{c}{\rightarrow} X$. Let $T: \ell_{1} \rightarrow X$ be an isomorphic embedding. Then $T^{*}: X^{*} \rightarrow \ell_{\infty}$ is unconditionally converging (since $c_{0} \hookrightarrow X^{*}$ ) and $T$ is not weakly precompact (since it is an isomorphism on $\ell_{1}$ ).

If $\ell_{1} \hookrightarrow X$, then every strongly bounded operator $T: C(K, X) \rightarrow Y$ is weakly precompact and has an unconditionally converging adjoint (by Corollary 2 and Theorem 12). This observation gives the following result.

COROLLARY 13. If $\ell_{1} \hookrightarrow X$, then every unconditionally converging (resp. completely continuous) operator $T: C(K, X) \rightarrow Y$ is weakly precompact and has an unconditionally converging adjoint.

Proof. If $T: C(K, X) \rightarrow Y$ is an unconditionally converging operator, then $T$ is strongly bounded. Since every completely continuous operator is unconditionally converging, every completely continuous operator $T: C(K, X) \rightarrow Y$ is strongly bounded.

THEOREM 14. Suppose that $\ell_{1} \stackrel{c}{\rightarrow} X$. Then every operator $T: C(K, X) \rightarrow Y$ has an unconditionally converging adjoint. 
Proof. Suppose $T: C(K, X) \rightarrow Y$ is an operator and $T^{*}: Y^{*} \rightarrow C(K, X)^{*}$ is not unconditionally converging. Using [9] or problem 8, p. 54, of [16], one obtains an isomorphic copy $U$ of $c_{0}$ in $Y^{*}$ on which $T^{*}$ acts as an isomorphism. If $L: c_{0} \rightarrow$ $U \subset Y^{*}$ is an isomorphic embedding, $T^{*} L: c_{0} \rightarrow C(K, X)^{*}$ is an isomorphism. Then $c_{0} \hookrightarrow C(K, X)^{*}$, and thus $\ell_{1} \stackrel{c}{\hookrightarrow} C(K, X)$ [9]. The main result in [29] implies that $\ell_{1} \stackrel{c}{\hookrightarrow} X$, a contradiction which concludes the proof.

The Banach space $X$ has property $\left(V^{*}\right)\left(\operatorname{resp} .\left(w V^{*}\right)\right)$ if every $V^{*}$-subset of $X$ is relatively weakly compact (resp. weakly precompact) $[\mathbf{1 0}, \mathbf{2 7}]$. The following result contains Theorem 1.6 of [22].

COROLlaRY 15. Suppose that $\ell_{1} \stackrel{c}{\leftrightarrow} X$ and $Y$ has property $\left(V^{*}\right)\left(\right.$ resp. $\left.\left(w V^{*}\right)\right)$. Then every operator $T: C(K, X) \rightarrow Y$ is weakly compact (resp. weakly precompact).

Proof. Suppose that $T: C(K, X) \rightarrow Y$ is an operator and $Y$ has property $\left(V^{*}\right)$ (resp. $\left.\left(w V^{*}\right)\right)$. By the previous result, $T^{*}: Y^{*} \rightarrow C(K, X)^{*}$ is unconditionally converging. Apply Theorem 3.10 of [24] to obtain that $T$ is weakly compact (resp. weakly precompact).

Corollary 2 of [6] shows that if $T^{*}: Y^{*} \rightarrow X^{*}$ is weakly precompact, then $T:$ $X \rightarrow Y$ is unconditionally converging and weakly precompact. It follows that if $T$ : $C(K, X) \rightarrow Y$ has a weakly precompact adjoint, then $T$ is strongly bounded (since it is unconditionally converging) and $T^{*}$ is unconditionally converging (by Theorem 12).

Suppose that $T: C(K, X) \rightarrow Y$ is an operator and $\hat{T}: B(\Sigma, X) \rightarrow Y^{* *}$ is its extension to $B(\Sigma, X)$. We remark that if $m \leftrightarrow T: C(K, X) \rightarrow Y$ is strongly bounded, then $m$ is $L(X, Y)$-valued [14] and $\hat{T}$ maps $B(\Sigma, X)$ into $Y$ (as noted in the Introduction).

TheOREM 16. Suppose that $T: C(K, X) \rightarrow Y$ is a strongly bounded operator. Then $T$ is weakly precompact if and only if its extension $\hat{T}: B(\Sigma, X) \rightarrow Y$ is weakly precompact.

Proof. Suppose that $T: C(K, X) \rightarrow Y$ is weakly precompact and $\hat{T}$ is not weakly precompact. Let $\epsilon>0, y^{*} \in B_{Y^{*}}$ and $\left(f_{n}\right)$ be a sequence in the unit ball of $B(\Sigma, X)$ such that $\left|\left\langle y^{*}, \hat{T}\left(f_{n}-f_{m}\right)\right\rangle\right|>\epsilon$, for $n \neq m$.

Using the existence of a control measure for $m$ and Lusin's theorem, one can find a compact subset $K_{0}$ of $K$ such that $\tilde{m}\left(K \backslash K_{0}\right)<\epsilon / 8$ and $g_{n}=\left.f_{n}\right|_{K_{0}}$ is continuous for each $n \in \mathbb{N}$. Let $H=\left[g_{n}\right]$ be the closed linear subspace spanned by $\left(g_{n}\right)$ in $C\left(K_{0}, X\right)$ and $S: H \rightarrow C(K, X)$ be the isometric extension operator given by Theorem 1 of [13]. If $h_{n}=S\left(g_{n}\right), n \in \mathbb{N}$, then $\left(h_{n}\right)$ is in the unit ball of $C(K, X)$, and for $n \neq m$,

$$
\begin{aligned}
\left|\left\langle y^{*}, T\left(h_{n}-h_{m}\right)\right\rangle\right| & \geq\left|\left\langle y^{*}, \int_{K_{0}}\left(h_{n}-h_{m}\right) d m\right\rangle\right|-\left|\left\langle y^{*}, \int_{K \backslash K_{0}}\left(h_{n}-h_{m}\right) d m\right\rangle\right| \\
& \geq\left|\left\langle y^{*}, \int_{K_{0}}\left(f_{n}-f_{m}\right) d m\right\rangle\right|-\epsilon / 4 \\
& \geq\left|\left\langle y^{*}, \int_{K}\left(f_{n}-f_{m}\right) d m\right\rangle\right|-\left|\left\langle y^{*}, \int_{K \backslash K_{0}}\left(f_{n}-f_{m}\right) d m\right\rangle\right|-\epsilon / 4 \\
& \geq\left|\left\langle y^{*}, \hat{T}\left(f_{n}-f_{m}\right)\right\rangle\right|-\epsilon / 2>\epsilon / 2 .
\end{aligned}
$$

This is a contradiction, since $T$ is weakly precompact. 
Corollary 17. Suppose that $m \leftrightarrow T: C(K, X) \rightarrow Y$ is a strongly bounded operator. If $T$ is weakly precompact, then $m(A): X \rightarrow Y$ is weakly precompact for each $A \in \Sigma$.

Proof. If $A \in \Sigma, A \neq \emptyset$, define $\theta_{A}: X \rightarrow B(\Sigma, X)$ by $\theta_{A}(x)=\chi_{A} x$. Then $\theta_{A}$ is an isomorphic isometric embedding of $X$ into $B(\Sigma, X)$ and $\hat{T} \theta_{A}=m(A)$. By Theorem $16, \hat{T}$ is weakly precompact, and thus $m(A)$ is weakly precompact.

A Banach space $X$ is a Grothendieck space if weak* and weak convergence of sequences in $X^{*}$ coincide.

Corollary 18. Suppose that $C(K)$ is a Grothendieck space. If $m \leftrightarrow T: C(K, X) \rightarrow$ $Y$ is a weakly precompact operator, then $m$ is $L(X, Y)$-valued and $m(A): X \rightarrow Y$ is weakly precompact for each $A \in \Sigma$.

Proof. Suppose $m \leftrightarrow T: C(K, X) \rightarrow Y$ is a weakly precompact operator. For each $x \in X$, define an operator $T_{x}: C(K) \rightarrow Y$ by $T_{x}(f)=T(f \cdot x), f \in C(K)$. Then $T_{x}$ is weakly precompact. By Corollary 6 of [6], $T_{x}^{*}$ is weakly precompact. Hence $T_{x}$ is an unconditionally converging operator on a $\mathrm{C}(\mathrm{K})$-space, and every unconditionally converging operator on a $\mathrm{C}(\mathrm{K})$-space is weakly compact $[14,27]$. If $m_{x}$ is the representing measure of $T_{x}$, then $m_{x}$ is $Y$-valued ([17], p. 105). Since $m_{x}(A)=$ $m(A) x, m$ is $L(X, Y)$-valued. An application of Corollary 6 of [1] concludes the proof.

If $S$ is a subspace of $X$ and $T: X \rightarrow Y$ is an operator, let $T_{S}$ denote the restriction of $T$ to $S$. A closed operator ideal $\mathcal{O}$ is said to be separably determined provided that for each pair of Banach spaces $X$ and $Y$, an operator $T: X \rightarrow Y$ belongs to $\mathcal{O}(X, Y)$ if and only if $T_{S} \in \mathcal{O}(S, Y)$ for each separable subspace $S$ of $X$.

THEOREM 19. Suppose that $T: C(K, X) \rightarrow Y$ is an operator and $\hat{T}: B(\Sigma, X) \rightarrow$ $Y^{* *}$ is its extension to $B(\Sigma, X)$. Then $T^{*}$ is weakly precompact if and only if $\hat{T}^{*}$ is weakly precompact.

Proof. If $\hat{T}^{*}$ is weakly precompact, then $\hat{T}$ is unconditionally converging and weakly precompact [6]. Hence $T$ is strongly bounded and $\hat{T}: B(\Sigma, X) \rightarrow Y$. Apply Theorem 4 of [6] to obtain a subspace $Z$ of $C(K, X)$ and an operator $S: Y \rightarrow \ell_{\infty}$ so that $S T(Z)=c_{0}$. Since $\hat{T}$ is an extension of $T$, there is a subspace $W$ of $B(\Sigma, X)$ so that $S \hat{T}(W)=c_{0}$. Thus by Theorem 4 of [6], $\hat{T}^{*}$ is not weakly precompact, and we have a contradiction.

Conversely, suppose that $T^{*}$ is weakly precompact. Then $T$ is strongly bounded and $\hat{T}: B(\Sigma, X) \rightarrow Y$. Let $\mathcal{O}=\left\{L: X \rightarrow Y \mid L^{*}\right.$ is weakly precompact $\}$. By Proposition 8 of [6], $\mathcal{O}$ is a closed separably determined operator ideal. Apply Proposition 4.1 of [2] to conclude that $\hat{T}$ is an element of $\mathcal{O}$, i.e. $\hat{T}^{*}$ is weakly precompact.

Corollary 20. ([6]) Suppose that $m \leftrightarrow T: C(K, X) \rightarrow Y$ is an operator. If $T^{*}$ is weakly precompact, then $m(A)^{*}: Y^{*} \rightarrow X^{*}$ is weakly precompact for each $A \in \Sigma$.

Proof. For $A \in \Sigma, A \neq \emptyset$, define $\theta_{A}: X \rightarrow B(\Sigma, X)$ by $\theta_{A}(x)=\chi_{A} x$. Then $\hat{T} \theta_{A}=$ $m(A), \hat{T}^{*}$ is weakly precompact (by Theorem 19), and thus $m(A)^{*}$ is weakly precompact. 


\section{REFERENCES}

1. C. Abbott, Weakly precompact and GSP operators on continuous function spaces, Bull. Polish Acad. Sci. Math. 37 (1989), 467-476.

2. C. Abott, E. Bator and P. Lewis, Strictly singular and cosingular operators on spaces of continuous functions, Math. Proc. Camb. Phil. Soc. 110 (1991), 505-521.

3. C. Abott, E. Bator, R. Bilyeu and P. Lewis, Weak precompactness, strong boundedness, and weak complete continuity, Math. Proc. Camb. Phil. Soc. 108 (1990), 325-335.

4. R. G. Bartle, A general bilinear vector integral, Studia Math. 15 (1956), 337-352.

5. E. Bator, P. Lewis and J. Ochoa, Evaluation maps, restriction maps, and compactness, Colloq. Math. 78 (1998), 1-17.

6. E. Bator and P. Lewis, Operators having weakly precompact adjoints, Math. Nachr. 157 (1992), 99-103.

7. J. Batt and E. J. Berg, Linear bounded transformations on the space of continuous functions, J. Funct. Anal. 4 (1969), 215-239.

8. C. F. Bello, On weakly compact and unconditionally converging operators in spaces of vector-valued functions, Revista Real Acad. Madrid 81 (1987), 693-706.

9. C. Bessaga, A. Pelczynski, On bases and unconditional convergence of series in Banach spaces, Studia Math. 17 (1958), 151-164. $109-120$.

11. F. Bombal and B. Porras, Strictly singular and strictly cosingular operators on $C(K, E)$, Math. Nachr. 143 (1989), 355-364.

12. J. Bourgain, An averaging result for $\ell_{1}$ sequences and applications to weakly conditionally compact sets in $L_{X}^{1}$, Israel J. Math. 32 (1979), 289-298.

13. F. Bombal, P. Cembranos, Characterizations of some classes of operators on spaces of vector-valued continuous functions, Math. Proc. Camb. Phil. Soc. 97 (1985), 137146.

14. J. K. Brooks and P. Lewis, Linear Operators and vector measures, Trans. Amer. Math. Soc. 192 (1974), 139-162.

15. P. Cembranos, N. Kalton, E. Saab and P. Saab, Pelczyinski's Property (V) on $C(\Omega, E)$ spaces, Math. Ann. 271 (1985), 91-97.

16. J. Diestel, Sequences and series in Banach spaces, Grad. texts in math., no. 92 (SpringerVerlag, Berlin, 1984).

17. J. Diestel and J. J. Uhl, Jr., Vector measures, math. surveys 15 (American Mathematical Society, Rhode Island, 1977.

18. N. Dinculeanu, Vector measures (Pergamon Press, Oxford, UK, 1967).

19. I. Dobrakov, On representation of linear operators on $C_{0}(T, X)$, Czechoslovak. Math. J. 21 (1971), 13-30.

20. N. Dunford and J.T. Schwartz, Linear operators. Part I: General theory (WileyInterscience, New Jersey, 1958).

21. G. Emmanuele, Another proof of a result of N. J. Kalton, E. Saab and P. Saab on the Dieudonné property in $C(K, E)$, Glasgow Math. J. 31 (1989), 137-140.

22. G. Emmanuele, On the Banach spaces with property $\left(V^{*}\right)$ of Pelczyinksi. II. Ann. Mat. Pura Appl. 160 (1991), 163-170. 285.

23. J. L. B. Gamlen, On a theorem of Pelczyinski, Proc. Amer. Math. Soc. 44 (1974), 283

24. I. Ghenciu and P. Lewis, Almost weakly compact operators, Bull. Polish. Acad. Sci. Math. 54 (2006), 237-256.

25. A. Grothendieck, Sur les applications linéaires faiblement compactes d'espaces du type $C(K)$, Canad. J. Math. 5 (1953), 129-173.

26. N. Kalton, E. Saab and P. Saab, On the Dieudonné property for $C(\Omega, E)$, Proc. Amer. Math. Soc. 96 (1986), 50-52.

27. A. Pelczyinski, Banach spaces on which every unconditionally converging operator is weakly compact, Bull. Acad. Polon. Sci. Math. Astronom. Phys. 10 (1962), 641-648.

28. C. Swartz, Unconditionally converging and Dunford-Pettis operators on $C_{X}(S)$, Studia Math. 57 (1976), 85-90. 
29. E. Saab and P. Saab, A stability property of Banach spaces not containing a complemented copy of $\ell_{1}$, Proc. Amer. Math. Soc. 84 (1982), $44-46$.

30. M. Talagrand, Weak Cauchy sequences in $L^{1}(E)$, Amer. J. Math. 106 (1984), 703 724.

31. M. Talagrand, La proprieté de Dunford-Pettis dans $C(K, E)$ et $L^{1}(E)$, Israel J. Math. 44 (1983), 317-321.

32. A. Ülger, Continuous linear operators on $C(K, X)$ and pointwise weakly precompact subsets of $C(K, X)$, Math. Proc. Camb. Phil. Soc. 111 (1992), 143-150. 